\title{
16 Referenzen
}

(Allianz 2010) Allianz der Wissenschaftsorganisationen: Neuregelung des Urheberrechts: Anliegen und Desiderate für einen Dritten Korb, 9.7.2010 - https://bit.ly/2Tz45wS.

(Allianz 2013) Allianz der deutschen Wissenschaftsorganisationen: Eine Handreichung für die parlamentarischen Beratungen über ein unabdingbares Zweitveröffentlichungsrecht https://bit.ly/2TpyiaR.

(Amini 2017) Seyavash Amini: Digitale Kultur zum Pauschaltarif? Anlass, Inhalt und Grenzen einer Vision für das Urheberrecht der Zukunft. Nomos.

(Axhamn 2019) Johan Axhamn: The new copyright directive: Collective licensing as a way to strike a fair balance between creator and user interests in copyright legislation - Kluwer Copyright Blog - https://bit.ly/2KhFvxf.

(Bahners 2017) Patrick Bahners: Der Volksmund weist den Weg. FAZ, Nr. 154:11, 6.7.2017 https://bit.ly/2H4zylb.

(Bannas/Sattar 2017) Günter Bannas; Majid Sattar: Große Politik im Talkshowsessel. FAZ. Politik, Nr. 147, 28.6.2017.

(Baron 2010) Benjamin Bajon: Interessenausgleich im Wissenschaftsurheberrecht? Wissenschaftsschranken nach dem „Zweiten Korb“ der Urheberrechtsreform “: Band 3 von Wissenschaftliche Schriften der WWU Münster, Reihe III. Verlagshaus Monsenstein und Vannerdat OHG - www.mv-wissenschaft.com.

(Bauer 2010) Christian Alexander Bauer: User generated content - Urheberrechtliche Zulässigkeit nutzergenerierter Medieninhalte. In: Henning Große Ruse-Khan; Nadine Klass; Silke von Lewinski (Hrsg.), Nutzergenerierte Inhalte als Gegenstand des Privatrechts. MPI Studies on Intellectual Property, Competition and Tax Law, 15. Springer.

(Becker 2019) Maximilian Becker: Von der Freiheit, rechtswidrig handeln zu können. ZUM, S. 636-648, 2019.

(Bendel 2019) Oliver Bendel: Wozu brauchen wir die Maschinenethik? In: Ders (Hrsg.), Handbuch Maschinenethik, S. 1-20. Springer Reference Geisteswissenschaften, 2019.

(Berger/Luckmann 1966/1969) Peter L. Berger; Thomas Luckmann: Die gesellschaftliche Konstruktion der Wirklichkeit. Eine Theorie der Wissenssoziologie. S. Fischer, 1966.

(Berger/Freyer 2016) Christian Berger; Simon Freyer: Neue individualvertragliche und kollektivrechtliche Instrumente zur Durchsetzung angemessener Urhebervergütungen. ZUM, S. 569-579, 2016.

(BOAI 2002) Budapest Open Access Initiative 2002 - https://bit.ly/20cCIEY.

(Bollier/Helfrich 2013) David Bollier; Silke Helfrich (Hrsg.): The wealth of the commons: $A$ world beyond market \& state. The Commons Strategies Group.

(2004b 2008) James Boyle: The public domain. Enclosing the commons of the mind. Yale University Press.

(Boyle 2003) James Boyle: The second enclosure movement and the construction of the public domain - https://bit.ly/2niStl2.

(Ilja Braun 2011) Ilja Braun: Raus aus den Regalen. iRights, 5. Dezember:2011 - https://bit.ly/ 2PgO2Fk.

(Brinkel 2006) Guido Brinkel: Filesharing. Verantwortlichkeit in Peer-to-Peer-Plattformen. Mohr Siebeck.

(Brinkmann 2018) Janis Brinkmann: Verlagspolitik in der Zeitungskrise: Theorien, Strukturen, Strategien. Nomos.

Ә Open Access. (C) 2020 Rainer Kuhlen (c))BY This work is licensed under a Creative Commons Attribution 4.0 License. https://doi.org/10.1515/9783110693447-016 
(Brock 2008) Ditmar Brock: Globalisierung: Wirtschaft - Politik - Kultur - Gesellschaft. VS Verlag für Sozialwissenschaften.

(Bruch/Pflüger 2014) Christoph Bruch; Thomas Pflüger: Das Zweitveröffentlichungsrecht des $\S 38$ Absatz 4 UrhG - Möglichkeiten und Grenzen bei der Anwendung in der Praxis. ZUM, S. 389-394, 2014 - https://bit.ly/2mbWDLI.

(Capurro 2003) Rafael Capurro: Informationsethik. Eine Standortbestimmung. International Review of Information Ethics, Nr. 1, Mai:2004 - https://bit.ly/36P5wN1.

(Chan et al. 2019) Leslie Chan; Angela Okune; Rebecca Hillyer; Denisse Albornoz; Alejandro Posada: Situating open science. University of Ottawa Press - https://bit.ly/2C0A5BC.

(Cole/Cole 1973) Jonathan R. Cole; Stephen Cole: Social stratification in science. Oxford Academics.

(Colledge/James 2015) Lisa Colledge; Christ James: A “basket of metrics"-the best support for understanding journal merit. European Science Editing, 41(3), August 2015 - https: //bit.ly/2nQAYJf.

(Cox 2015) Krista L. Cox (Association of Research Libraries - ARL: Issue brief): Text and data mining and fair use in the United States - https://bit.ly/1LVQyp8.

(Cox 2012) Krista L. Cox: United States four fair use factors and the WTO Three-Step Test. Knowledge Ecology International, 20.11.2012) - https://bit.ly/2t4Jyaw.

(Crewes 2010) Kenneth D. Crews: Copyright law and distance education: Overview of the TEACH Act, 17.8.2010 - https://bit.ly/2nk8Z4jf.

(Cronin 2001) Blaise Cronin: Hyperauthorship: A postmodern perversion or evidence of a structural shift in scholarly communication practices? Journal of the American Society for Information Science and Technology, 52:558-569, 2001.

(Czepel 2015) Robert Czepel: Fünf Konzerne regieren die Wissenschaft. Science ORF, 11.6.2015 - https://bit.ly/2kArkJr.

(dbv 2017) Stellungnahme des Deutschen Bibliotheksverbands e. V. zum „Entwurf eines Gesetzes zur Angleichung des Urheberrechts an die aktuellen Erfordernisse der Wissensgesellschaft des (Urheberrechts-Wissensgesellschafts-Gesetz - UrhWissG)“ des BMJV vom 22.2.2017 - https://bit.ly/2Np1q7J.

(Dinleavy 2018) Patrick Dinleavy: Open Access knowledge. Digital style guide. Writing for Research, 8.1.2018 - https://bit.ly/2CZoe93.

(Drees 2016) Bastian Drees: Text und Data Mining: Herausforderungen und Möglichkeiten für Bibliotheken. Perspektive Bibliothek, 5.1:49-73 - https://bit.ly/2k5znhc.

(Duppelfeld 2014) Monika Duppelfeld: Das Urheberrecht der Bibliotheken im Informationszeitalter. Geistiges Eigentum und Wettbewerbsrecht 97. Mohr Siebeck.

(de la Durantaye 2016) Katharina de la Durantaye: Die Bildungs- und Wissenschaftsschranke Warum kurz springen? ZUM, S. 475-481, 2016.

(de la Durantaye 2014b) Katharina de la Durantaye: Stellungnahme zu dem Entwurf eines Gesetzes zur Nutzung verwaister und vergriffener Werke und einer weiteren Änderung des Urheberrechtsgesetzes (BT-Drucks. 17/13423) - https://bit.ly/2njQRHL.

(de la Durantaye 2014a) Katharina de la Durantaye: Allgemeine Bildungs- und Wissenschaftsschranke. Verlagshaus Monsenstein und Vannerdat - https://bit.ly/2mkGZxm.

(de la Durantaye 2005) Katharina de la Durantaye: Der Schutz literarischer Urheberschaft im Rom der klassischen Antike. Mohr Siebeck - Dissertation, Humboldt-Universität zu Berlin 2003.

(de la Durantaye/Kuschel 2019) Katharina de la Durantaye; Linda Kuschel: Vergriffene Werke größer gedacht: Art. 8-11 DSM-Richtlinie. ZUM, S. 694-703, 2019. 
(Depenheuer/Peifer 2008) Otto Depenheuer; Karl-Nikolaus Peifer Geistiges Eigentum: Schutzrecht oder Ausbeutungstitel. Zustand und Entwicklungen im Zeitalter von Digitalisierung und Globalisierung. Springer.

(Dreier 2019a) Thomas Dreier: Die Schlacht ist geschlagen - ein Überblick. Zum Ergebnis des Copyright Package der EU-Kommission. GRUR, 8:771-779, 2019.

(Dreier 2019b) Thomas Dreier: Der Schrankenkatalog: Adäquate Zugangsregeln für die Wissensgesellschaft? ZUM, S. 384-393.

(Dreier/Hilty 2015) Thomas Dreier; Reto M. Hilty: Vom Magnettonband zu Social Media. Festschrift. 50 Jahre Urheberrechtsgesetz (UrhG). . C. H. Beck Verlag.

(Dreier/Schulze 2018) Thomas Dreier; Gernot Schulze: Urheberrechtsgesetz: UrhG. C. H. Beck.

(Enquete-Kommission 2011) Enquete-Kommission „Internet und digitale Gesellschaft“: Dritter Zwischenbericht Urheberrecht. Drucksache Deutscher Bundestag 17/7899, 23.11.2011 https://bit.ly/2mbZiFc.

(Felber 2019) Christian Felber: This not economy. Aufruf zur Revolution der Wirtschaftswissenschaft. Deuticke.

(Felber 2018) Christian Felber: Gemeinwohlökonomie. Piper.

(Forst/Günther 2011) Rainer Forst; Klaus Günther (Hrsg.): Die Herausbildung normativer Ordnungen. Interdisziplinäre Perspektiven. Campus Verlag - (vgl. dazu Harald G. Kratochvila: Rezension von (Forst/Günther 2011) vom 7.7.2011 - https://bit.ly/2mbu40x).

(Förster 2008) Achim Förster: Fair Use: ein Systemvergleich der Schrankengeneralklausel des US-amerikanischen Copyright Act mit dem Schrankenkatalog des deutschen Urheberrechtsgesetzes. Mohr Siebeck.

(Fyfe et al. 2017) Aileen Fyfe; Kelly Coate; Stephen Curry; Stuart Lawson; Noah Moxham; Camilla Mørk Røstvik: Untangling academic publishing: A history of the relationship between commercial interests, academic prestige and the circulation of research https://bit.ly/2kzKFuh.

(Geiger 2006) Christoph Geiger: The Three-step test, a thread to a balanced copyright law? IIC - International Review of Intellectual Property and Competition Law, 37(6):683-699, Jan 2006 - https://bit.ly/2ndagdy.

(Geiger et al. 2010) Christophe Geiger; Reto Hilty; Jonathan Griffiths; Uma Suthersanen: Declaration - A balanced interpretation of the "Three-step test". Copyright Law, 1. JIPITEC 119 para:1, 2010 - https://bit.ly/2nhzTdq.

(Geiger et al. 2013) Christoph Geiger; Daniel Gervais; Martin Senftleben: The three-step-test revisited. How to use the test's flexibility in national copyright law. PIJIP Research Paper Nr. 2013-14 - https://bit.ly/2ml7XVL.

(Gelke 2013) Erik Gelke: Mashups im Urheberrecht: Band 269 von Schriftenreihe des Archivs für Urheber- und Medienrecht. Nomos - https://bit.ly/2nhUmys.

(Gervais 2011) Daniel Gervais: The landscape of collective management schemes. Columbia Journal of Law \& the Arts, 34(4); über SSRN: https://bit.ly/2Mkpiau.

(Gervais 2003) Daniel Gervais: Application of an extended collective licensing regime in Canada: Principles and issues related to implementation. Vanderbilt Public Law Research Paper Nr. 11-26 - SSRN: https://bit.ly/2oiRjqP.

(Gilcher-Holtey 2005) Ingrid Gilcher-Holtey (Hrsg.): Zwischen den Fronten. Positionskämpfe europäischer Intellektueller im 20. Jahrhundert. Akademie Verlag.

(Griffiths 2009) Jonathan Griffiths: The 'three-step test' in European Copyright Law - Problems and solutions (22.9.2009). Queen Mary School of Law Legal Studies Research Paper Nr. 31/2009 - SSRN: https://bit.ly/2ml6lLH. 
(Grisse 2018) Karina Grisse: Internetangebotssperren. Zivilrechtliche Vermittlerhaftung von Internetzugangsanbietern. Mohr Siebeck.

(Grötschel 2016) Martin Grötschel: Elektronisches Publizieren, Open Access, Open Science und ähnliche Träume. Preprint: https://bit.ly/2xWyiv2.

(Grötschel/Lügger 1996) Martin Grötschel; Joachim Lügger: Wissenschaftliche Information und Kommunikation im Umbruch. Über das Internet zu neuen wissenschaftlichen Informationsinfrastrukturen. Forschung \& Lehre, Heft 1/1996:194-198 - https://bit.ly/3812mGl.

(Grünberger 2018a) Michael Grünberger: Die Entwicklung des Urheberrechts im Jahr 2017. Teil I. ZUM, S. 271-285, 2018.

(Grünberger 2018b) Michael Grünberger: Die Entwicklung des Urheberrechts im Jahr 2017. Teil II. ZUM, S. 321-340, 2018.

(Grünberger 2016) Michael Grünberger: Die Bildungs- und Wissenschaftsschranke - Ein angemessener Interessenausgleich? ZUM, S. 473-474, 2016.

(GRUR) Stellungnahme der GRUR zum Referenten-Entwurf eines Gesetzes zur Nutzung verwaister Werke und zu weiteren Änderungen des Urheberrechtsgesetzes und des Urheberrechtswahrnehmungsgesetzes vom 20.2.2013 - https://bit.ly/2suD4Bq.

(Guretzky o. J.) Bernhard von Guretzky: Wissensethik - https://bit.ly/2mbkivP.

(Gutknecht 2015) Christian Gutknecht: Zahlungen der ETH Zürich an Elsevier, Springer und Wiley nun öffentlich. wisspub.net - Gemeinschaftsblog zu wissenschaftlicher Kommunikation im Netz, 29.8.2015 - https://bit.ly/1QiDgUS.

(Hanrider 2008) Tine Hanrider: Moralische Argumente in den Internationalen Beziehungen. Grenzen einer verständigungstheoretischen »Erklärung « moralischer Debatten. Zeitschrift für Internationale Beziehungen. Archiv, 2008 - https://bit.ly/33fZAuS.

(Hanrider 2009) Tine Hanrider: Die Rechtfertigung des Urheberrechts unter besonderer Berücksichtigung des Nutzerschutzes: Band 53 von Abhandlungen zum Urheber- und Kommunikationsrecht. Nomos - https://bit.ly/2nStV2Q.

(Hansen 2005) Gerd Hansen: Zugang zu wissenschaftlicher Information - alternative urheberrechtliche Ansätze. GRUR Int., S. 378-388, 2005.

(Hargreaves/Hugenholtz 2013) Ian Hargreaves; P. Bernt Hugenholtz: Copyright reform for growth and jobs. Modernising the European Copyright Framework - https://bit.ly/2JqJ82W.

(Hargreaves 2011) Ian Hargreaves: Digital Opportunity. A Review of Intellectual Property and Growth. UK Department for Business, Innovation \& Skills (seit 2016: Department for Business, Energy and Industrial Strategy (BEIS) - https://bit.ly/1/2QFl3.

(Hartmann 2017) Thomas Hartmann: Zwang zum Open Access-Publizieren? Der rechtliche Präzedenzfall ist schon da! LIBREAS. Library Ideas, 32 - https://bit.ly/2nezBUB.

(Haucap et al. 2016) Justus Haucap; Ina Loebert; Gerald Spindler; Susanne Thorwarth: Ökonomische Auswirkungen einer Bildungs- und Wissenschaftsschranke im Urheberrecht (Studie im Auftrag des BMBF). Düsseldorf University Press - https://bit.ly/2b0ZQ7M.

(Hauff-Hartig 2018) Stefan Hauff-Hartig: Fehl-, Falsch- und Desinformation aus dem Blickwinkel der Informationswissenschaften. Simon Verlag.

(Hausmanninger/Capurro 2002) Thomas Hausmanninger; Rafael Capurro (Hrsg.): Netzethik. Grundlegungsfragen der Internetethik: Band 1 von Schriftenreihe des ICIE: S. 13-36. Fink.

(Heckmann/Weber 2006) Jörn Heckmann; Marc Philipp Weber: Open Access in der Informationsgesellschaft - $\S 38$ de lege ferenda UrhG. GRUR Int., Heft 12, 2006.

(Heine/Schafdecker 2018) Robert Heine; Julia Schafdecker: Die Maschine als Urheber? Legal Tribune Online, 1.12.2018 - https://bit.ly/3a3JhFq. 
(Helfrich/Bollier 2019) Silke Helfrich; David Bollier: Frei, fair und lebendig - Die Macht der Commons. transcript - https://bit.ly/2mbwiNb.

(Helfrich/HBS 2012) Silke Helfrich; Heinrich-Böll-Stiftung (Hrsg.): Commons. Für eine neue Politik jenseits von Markt und Staat. transcript.

(Helfrich/HBS 2009) Silke Helfrich; Heinrich-Böll-Stiftung (Hrsg.): Wem gehört die Welt. Zur Wiederentdeckung der Gemeingüter. Oekom - https://bit.ly/2TyeZTA.

(Herb 2019) Ulrich Herb: Überwachungskapitalismus und Wissenschaftssteuerung. TELEPOLIS, 29. Juli:2019 - https://bit.ly/385rMme.

(Herb 2018) Ulrich Herb: Zwangsehen und Bastarde. Wohin steuert Big Data die Wissenschaft? Information, Wissenschaft \& Praxis, 69(2-3):81 ff., 2018.

(Hess/Ostrom 2007) Charlotte Hess; Elinor Ostrom: Understanding knowledge as a commons. From Theory to practice. The MIT Press.

(Hevers 2015) Erik Hevers: Informationszugangsansprüche des forschenden Wissenschaftlers. Duncker \& Humblot.

(Hilf/Severiens 2013) Eberhard R. Hilf; Thomas Severiens: Vom Open Access für Dokumente und Daten zu open content in der Wissenschaft. In: Rainer Kuhlen; Wolfgang Semar; Dietmar Strauch (Hrsg.), Handbuch Grundlagen von Information und Dokumentation, S. 379-395. De Gruyter, 6. Aufl.

(Hilty 2007) Reto M. Hilty: Das Urheberrecht darf nicht zu einem Instrument gegen die Wissenschaft ausarten. Süddeutsche Zeitung, 12.4.2007:18.

(Hilty 2006) Reto M. Hilty: Das Urheberrecht und der Wissenschaftler. GRUR Int., S. 179-181, 2006.

(Hilty/Klass 2009) Reto M. Hilty; Nadine Klass: Stellungnahme des Max-Planck-Instituts für Geistiges Eigentum, Wettbewerbs- und Steuerrecht zur Anfrage des Bundesministeriums der Justiz vom 19. Februar 2009 - https://bit.ly/2lEU7x3.

(Hilty/Richter 2019) Reto Hilty; Heiko Richter: Vom Drang, Freiheit zu regulieren. Max Planck Forschung, 3/19 - https://bit.ly/2NoM7LB.

(Hilty/Richter 2017) Reto Hilty; Heiko Richter: Position Statement of the Max Planck Institute for Innovation and Competition on the Proposed Modernisation of European Copyright Rules Part B Exceptions and Limitations. (Art. 3 - Text and Data Mining), 14.01.2017 https://bit.ly/2nhDLL8.

(Höpfner/Amschewitz 2019) Clemens Höpfner; Dennis Amschewitz: Die Zweitveröffentlichungspflicht im Spannungsfeld von Open-Access-Kultur und Urheberrecht. NJW, S. 29662973, 2019.

(Hoeren/Neubauer 2012) Thomas Hoeren; Arne Neubauer: Zur Nutzung urheberrechtlich geschützter Werke in Hochschulen und Bibliotheken. ZUM, 8/9:636-643, 2012.

(Hoeren/Köcher 2005) Thomas Hoeren; Jan K. Köcher: Der Wissenschaftler als Autor - Überlegungen zum Referentenentwurf .... In: Ulrich Sieber; Thomas Hoeren (Hrsg.), Urheberrecht für Bildung und Wissenschaft - Anforderungen an das Zweite Gesetz zur Regelung des Urheberrechts in der Informationsgesellschaft, Beiträge zur Hochschulpolitik 2/2005, S. 12-18https://bit.ly/2mnSofq.

(Hoeren 2019) Thomas Hoeren: Datenbesitz statt Dateneigentum. Erste Ansätze zur Neuausrichtung der Diskussion um die Zuordnung von Daten. MMR, S. 5-8, 2019.

(Hoeren 2018) Thomas Hoeren: Das Urheberrechts-Wissensgesellschafts-Gesetz Ein richtiger Schritt für das Urheberrecht in Bildung und Forschung? Zeitschrift für Internationales Wirtschaftsrecht (IWRZ), 3/2018. 
(Hoeren 2011) Thomas Hoeren: Kleine Werke? - Zur Reichweite von § 52 a UrhG. ZUM, 5/2011:369-375.

(Hoeren 2004) Thomas Hoeren: Informationsgerechtigkeit als Leitperspektive des Informationsrecht. In: Jürgen Taeger; Andreas Wiebe (Hrsg.), Informatik - Wirtschaft - Recht: Regulierung in der Wissensgesellschaft. Festschrift für Wolfgang Kilian zum 65. Geburtstag, S. 91-102. Nomos.

(Hoeren/Kalberg 2006) Thomas Hoeren; N. Kalberg: Der amerikanische TEACH Act und die deutsche Schrankenregelung zur „Öffentlichen Zugänglichmachung für Unterricht und Forschung“ (§52a UrhG) im Vergleich. ZUM, 8/9/2006:600-604.

(Höffner 2010) Eckhard Höffner: Geschichte und Wesen des Urheberrechts. 2 Bände. Verlag Europäische Wirtschaft.

(Hornbostel 1997) Stefan Hornbostel: Wissenschaftsindikatoren: Bewertungen in der Wissenschaft. Westdt. Verlag - https://bit.ly/2lFdsya.

(Hugenholtz et al. 2006) Bernt Hugenholtz et al.: The recasting of copyright \& related rights for the knowledge economy. Institute for Information Law University of Amsterdam The Netherlands - https://bit.ly/2VGlsi2.

(Hugenholtz/Okediji 2007) P. Bernt Hugenholtz; Ruth L. Okediji: Conceiving an international instrument on Limitations and exceptions to copyright. Endbericht, 7.3.2007 - https://bit.ly/ 2nSzRc7.

(IFLA 2015) IFLA critiques licensing solutions at the European Parliament, 7.5.2015 - https: //bit.ly/2Zpj40S.

(Jaki 2019) Bernhard Jaki: Das Recht der Künstlichen Intelligenz. Möglichkeiten und Grenzen zivilrechtlicher Regulierung. MMR, S. $711 \mathrm{ff.}, 2019$.

(Jani 2016) Ole Jani: Die Bildungs- und Wissenschaftsschranke - Der Gesetzgeber muss erklären, was das Ziel einer solchen Schranke sein soll. ZUM, S. 481-483, 2016.

(Jänich 2002) Volker Jänich: Geistiges Eigentum - eine Komplementärerscheinung zum Eigentum? Mohr Siebeck.

(Jiang/Gervais 2012) Fuxiao Jiang; Daniel Gervais: Collective management organizations in China: Practice, problems and possible solutions. The Journal of World Intellectual Property, 15(3):221-237 - SSRN.

(Jütte 2019) Bernd Justin Jütte: The new copyright directive: Digital and cross-border teaching exception (article 5). Kluwer Coypright Blog - https://bit.ly/205hQbb.

(Kant 2002) Horst Kant: Disziplinäre Gesellschaften als Träger von Fachzeitschriften. Einige Anmerkungen zur Entstehung physikalischer Zeitschriften im 19. Jahrhundert in Deutschland: In: (Parthey/Umstätter 2002), S. $61 \mathrm{ff.}$

(Karpinski 2015) Adam Karpinski: Creating a copyright exception for education. Communia, 3.11.2015 - https://bit.ly/3abJl63.

(Kerres/Jechle 2001) Michael Kerres; Thomas Jechle: Didaktische Konzeption des Tele-Lernens. In: L. J. Issing; P. Klimsa (Hrsg.), Information und Lernen mit Multimedia. Beltz, 2. Aufl.

(Kirchmaier 2017) Robert Kirchmaier: Das Urheberrechts-Wissensgesellschafts-Gesetz und seine Auswirkungen auf den Bereich der Kultureinrichtungen. Kultur und Recht, 19:3-4 https://bit.ly/2nTuOYZ.

(Kirsch-Händert 1989) Johannes Kirsch-Händert: Zeitgeist - Die Vermittlung des Geistes mit der Zeit: Eine wissenssoziologische Untersuchung zur Geschichtsphilosophie Hans Blumenbergs. Europäische Hochschulschriften. Sociologie, 182.

(Kohler 1907) Josef Kohler: Urheberrecht an Schriftwerken und Verlagsrecht. Verlag F. Enke.

(Konersmann 2015) Ralf Konersmann: Die Unruhe der Welt. S. Fischer Wissenschaft. 
(Konersmann 2004) Ralf Konersmann: Zeitgeist. In: Joachim Ritter; Karlfried Gründer; Gottfried Gabriel (Hrsg.), Historisches Wörterbuch der Philosophie, Band 12 (W-Z). Schwabe AG.

(Koroch 2016) Stefan Koroch: Das Leistungsschutzrecht des Presseverlegers. Mohr Siebeck.

(Krempl 2018) Stefan Krempl: US-Kongress erwägt Copyright-Verlängerung auf bis zu 144 Jahre. Heise online, 19.05.2018 - https://bit.ly/2m0uRBa.

(Krempl 2007) Stefan Krempl: Neues Urheberrecht tritt Anfang 2008 in Kraft. Heise online, 1.11.2007 - https://bit.ly/2mnkUOH.

(Kretschmer 2010) Michael Kretschmer: Wo geforscht wird, fallen Früchte: Open access. Freier Zugang sollte zur Vorbedingung öffentlicher Förderung werden. FAZ, 23.6.2010.

(Kreutzer 2008) Till Kreutzer: Das Modell des deutschen Urheberrechts und Regelungsalternativen. Konzeptionelle Überlegungen zu Werkbegriff, Zuordnung, Umfang und Dauer des Urheberrechts als Reaktion auf den urheberrechtlichen Funktionswandel. Nomos.

(Kreutzer 1999) Till Kreutzer: Die Entwicklung des Urheberrechts in Bezug auf Multimedia der Jahre 1994-1998. Arbeitspapiere des Hans-Bredow-Instituts, Nr. 3 - https://bit.ly/2mlilw).

(Kreutzer/Lahmann 2019) Till Kreutzer; Henning Lahmann: Rechtsfragen bei Open Science. Hamburg University Press - https://bit.ly/2vzgUPJ.

(Kreutzer/Hirche 2017) Till Kreutzer; Tom Hirche: Rechtsfragen zur Digitalisierung in der Lehre.

(Krüger/Dettling 2019) Heinz-Uwe Dettling; Stefan Krüger: Erste Schritte im Recht der Künstlichen Intelligenz. Entwurf der „Ethik-Leitlinien für eine vertrauenswürdige KI“. MMR, S. 211-217, 2019.

(Kuhlen 2017b) Rainer Kuhlen: UrhWissG - das neue Wissenschaftsurheberrecht bleibt regulierungstechnisch überspezifisch problematisch. Information, Wissenschaft \& Praxis, 68(4):227-245, 2017 - https://bit.ly/2nj9FH6.

(Kuhlen 2017a) Rainer Kuhlen: Zum Vorschlag der EU-Kommission für eine neue Urheberrechtsrichtlinie. Information, Wissenschaft \& Praxis, 68(2-3):139-153, 2017.

(Kuhlen 2016) Rainer Kuhlen: Der Heizer sollte nicht auf der E-Lok bleiben - Die Allgemeine Bildungs- und Wissenschaftsschranke ist nötig und möglich. ZUM, S. 507-513, 2016.

(Kuhlen 2015b) Rainer Kuhlen: Wie umfassend soll/darf /muss sie sein, die allgemeine Bildungs- und Wissenschaftsschranke? Zeitschrift für Geistiges Eigentum / Intellectual Property Journal, 7(1):77-125 - (Autortext: https://bit.ly/2lKvcbCf).

(Kuhlen 2015a) Rainer Kuhlen: Kopernikanische Wende in der EU-Urheberrechtsdebatte? Die Politik in Deutschland sollte das nutzen. Netzpolitik.org, 21.1.2015.

(Kuhlen 2014b) Rainer Kuhlen: Copyright and information ethics - an insight. In: Amélie Vallotton; Hermann Rösch; Christoph Stückelberger (Hrsg.), Ethical Dilemmas in the Information Society. Codes of Ethics for Librarian and Archivists, Papers from the IFLA/FAIFE Satellite Meeting, S. 191-200.

(Kuhlen 2014a) Rainer Kuhlen: Interdependenzen zwischen Informationsethik und politischem Handeln - am Beispiel einer kontroversen Urheberrechtsregulierung (Zweitverwertungsrecht). Zeitschrift für Politikwissenschaft (ZPol), Sonderband Ethik und Politikmanagement, S. 193-221 - Preprint, https://bit.ly/2nhNotg.

(Kuhlen 2013c) Rainer Kuhlen: Information, Informationswissenschaft. Artikel A1. In: Rainer Kuhlen; Wolfgang Semar; Dietmar Strauch (Hrsg.), Handbuch Grundlagen von Information und Dokumentation, S. 1-24. De Gruyter, 6. Aufl.

(Kuhlen 2013b) Rainer Kuhlen: Manifestierung der Dreiklassengesellschaft. Spectrum.de, 19.9.2013 - https://bit.ly/2nf4Qia. 
(Kuhlen 2013a) Rainer Kuhlen: Erfolgreiches Scheitern revisited - in Richtung einer allgemeinen Wissenschaftsklausel. Bibliothek, Forschung und Praxis, 37(1):1-10 - https: //bit.ly/2naBaCR.

(Kuhlen 2012c) Rainer Kuhlen: Knowledge is the water of the mind. How to structure rights in immaterial commons. In: David Bollier; Silke Helfrich (Hrsg.), The wealth of the commons. A world beyond market \& state, S. 331-339. The Commons Strategies Group (www.wealthofthecommons.org).

(Kuhlen 2012b) Rainer Kuhlen: Wissensökonomie und Wissensökologie zusammen denken. In: Silke Helfrich; Heinrich-Böll-Stiftung (Hrsg.), Commons. Für eine neue Politik jenseits von Markt und Staat, S. 405-413. transcript - https://bit.ly/21MeEzv<9.

(Kuhlen 2012a) Rainer Kuhlen: Was haben Eigentum und Wissenschaftsfreiheit mit dem Urheberrecht zu tun? Mit Reförmchen ist es nicht länger getan. FiFF-Kommunikation, 1/2013:48-52 - https://bit.ly/2lF16WL.

(Kuhlen 2011b) Rainer Kuhlen: Richtungsweisend oder eine verpasste Chance? Der Copyright-Code des Wittem Projekts. Information Technology and E-Commerce Law (JIPITEC), 2/2011:18-25.

(Kuhlen 2011a) Rainer Kuhlen: Der Streit um die Regelung des Zweitveröffentlichungsrechts im Urheberrecht - oder: Was macht Wissenschaftsfreiheit aus? In: Joachim Griesbaum; Thomas Mandl; Christa Womser-Hacker (Hrsg.), Information und Wissen: global, sozial und frei? ISI 2011 - 12. Internationales Symposium für Informationswissenschaft, Universität Hildesheim, 9.-11. März 2011 - Preprint: https://bit.ly/2nS7f2K.

(Kuhlen 2010d) Rainer Kuhlen: Kommentar zu Steinhauer: Das Recht auf Sichtbarkeit - https: //bit.ly/2MvsO4h - ausführlich: Ein Recht auf Sichtbarkeit - aber nicht auch ein Recht auf Sichtbarwerden?

(Kuhlen 2010c) Rainer Kuhlen: Ethical foundation of knowledge as a common. In: Proceedings of the International Conference commemorating the 40th Anniversary of the Korean Society for Library and Information Science, Seoul, 8.10.2010 - https://bit.ly/2kgWZiZ.

(Kuhlen 2010b) Rainer Kuhlen: In Richtung einer allgemeinen Wissenschaftsklausel. Information - Wissenschaft und Praxis (IWP), 8/2010:427-434 - http://bit.ly/cZyGNW.

(Kuhlen 2010a) Rainer Kuhlen: Freier Zugang zu den verwaisten Werken. Kann der freie Zugang zu einem gewichtigen Teil des kulturellen Erbes, zu den verwaisten Werken und damit zu einem gewichtigen Teil des Commons, in Europa bald Wirklichkeit werden? - https: //bit.ly/2mlEk6I.

(Kuhlen 2008) Rainer Kuhlen: Erfolgreiches Scheitern - eine Götterdämmerung des Urheberrechts? vwh Verlag Werner Hülsbusch - https://bit.ly/2mnpD2V.

(Kuhlen 2004f) Rainer Kuhlen: Wissensökologie. In: Rainer Kuhlen; Wolfgang Semar; Dietmar Strauch (Hrsg.), Handbuch Grundlagen von Information und Dokumentation, S. 105-113. Saur Verlag, 5. Aufl. - https://bit.ly/2ITmEyU.

(Kuhlen 2004e) Rainer Kuhlen: Nachhaltigkeit muss nicht Verknappung bedeuten - in Richtung Wissensökologie. Sonderausgabe der Zeitschrift des Forums InformatikerInnen für Frieden und gesellschaftliche Verantwortung, über „IT und Nachhaltigkeit“, 21(4):15-19 https://bit.ly/2kzzSAB.

(Kuhlen 2004d) Rainer Kuhlen: Informationsethik. In: Rainer Kuhlen; Wolfgang Semar; Dietmar Strauch (Hrsg.), Handbuch Grundlagen von Information und Dokumentation, S. 61-71. Saur Verlag, 5. Aufl. - https://bit.ly/2kx3m1X.

(Kuhlen 2004c) Rainer Kuhlen: Informationsethik - Umgang mit Wissen und Information in elektronischen Räumen. Reihe UTB 2454. Universitätsverlag Konstanz (UVK). 
(Kuhlen 2004b) Rainer Kuhlen: Wem gehört die Information im 21. Jahrhundert - eine Skizze. In: A. Büllesbach; T. Dreier (Hrsg.), Wem gehört die Information im 21. Jahrhundert? Proprietäre versus nicht proprietäre Verwertung digitaler Inhalte, S. 1-9. Verlag Dr. Otto Schmidt https://bit.ly/2nf9QDs.

(Kuhlen 2004a) Rainer Kuhlen: Kollaboratives Schreiben. Wenn Autoren und ihre Werke Kollaborateure werden - was ändert sich dann? Oder: wenn Kommunikation ein Recht, gar ein Menschenrecht wird - was ändert sich dann? In: C. Bieber; C. Leggewie (Hrsg.), Interaktivität ein transdisziplinärer Schlüsselbegriff, S. 216-239. Campus-Verlag - https: //bit.ly/2nUlUup.

(Kuhlen 1999) Rainer Kuhlen: Die Konsequenzen der Informationsassistenten. Was bedeutet informationelle Autonomie oder wie kann Vertrauen in elektronische Dienste in offenen Informationsmärkten gesichert werden? Nr. stw 1443 in Suhrkamp Taschenbuch wissenschaft. Suhrkamp-Verlag - https://bit.ly/2IKDj84.

(Kuhlen/Brüning 2004) Rainer Kuhlen; Jochen Brüning: Creative Commons (CC) - für informationelle Selbstbestimmung, gegen den Trend des Urheberrechts/Copyright als Handelsrecht oder: Chancen für einen innovativen Drei-Stufen-Test? Information, Wissenschaft \& Praxis (IWP), 8/2004:449-454 - https://bit.ly/2lBvt0e.

(Lakoff/Johnson 2011) George Lakoff; Mark Johnson: Leben in Metaphern: Konstruktion und Gebrauch von Sprachbildern. Springer.

(Lalé 2017) Céline Lalé: Reform mit Ausnahme: Ein unnötiges Geschenk für Zeitungsverlage. iRIGHTS info, 3.7.2017 - https://bit.ly/2uNVbgZ.

(Landow 1992) George P. Landow: Hypertext. The convergence of contemporary critical theory and technology. John Hopkins University Press.

(Larivière et al. 2015) Vincent Larivière; Stefanie Haustein; Philippe Mongeon: The Oligopoly of Academic Publishers in the Digital Era. PLOS ONE, 10.6.2015 - https://bit.ly/2FPXyKz.

(Leistner 2008) Matthias Leistner: Buchrezension zu (Kreutzer 2008). JIPITEC I, 2:165 - para. 1.

(Leistner/Dreier 2013) Matthias Leistner; Thomas Dreier: Urheberrecht im Internet: die Forschungsherausforderungen. GRUR, S. 881-892, 2013.

(Lerner/Lin 2012) Josh Lerner; Eric Lin: Collaboration in intellectual property: an overview. WIPO, 6/2012.

(Leventer 2012) N. Orly Leventer: Google Book Search und vergleichendes Urheberrecht. Unter Heranziehung des deutschen und US-amerikanischen Rechts. Nomos - (Dissertation, Universität Freiburg 2011).

(Lessig 1999/2006) Lawrence Lessig: Code and other laws of cyberspace. Basic Books.

(Lipinski 2005) Thomas A. Lipinski: Copyright Law and the Distance Education Classroom. Scarecrow Press - https://bit.ly/2ko2Jrv.

(Luhmann 1984/1987) Niklas Luhmann: Soziale Systeme. Grundriss einer allgemeinen Theorie - suhrkamp taschenbuch wissenschaft.

(Maas 2016) Heiko Maas: Kulturelle Werke - mehr als nur ein Wirtschaftsgut. Keynote auf der Konferenz „Die Zukunft des Urheberrechts - 50 Jahre Urheberrecht in Deutschland“ am 1. Dezember 2015 in Berlin. ZUM, S. 207-211, 2016.

(Maier 2003) Matthias Leonhard Maier: Wissens- und ideenorientierte Ansätze in der Politikwissenschaft: Versuch einer systematischen Übersicht. In: Matthias Leonhard Maier; Frank Nullmeier; Tanja Pritzlaff; Achim Wiesen (Hrsg.), Politik als Lernprozess. Wissenszentrierte Ansätze der Politikanalyse. Springer.

(Marl 2017) Johannes Marl: Der Begriff der Öffentlichkeit im Urheberrecht. Mohr Siebeck. 
(Menard 2012) Michael Menard: Urheber: Filter oder Schöpfer? boersenblatt.net, 5.7.2012 https://bit.ly/2YQ4ekq.

(Menne-Haritz 2013) Angelika Menne-Haritz: D 10 Archive. In: Grundlagen der praktischen Information und Dokumentation. In: Rainer Kuhlen; Wolfgang Semar; Dietmar Strauch (Hrsg.), Handbuch Grundlagen von Information und Dokumentation, S. 596-608. Saur Verlag/De Gruyter, 6. Aufl.

(Metzger 2002) Axel Metzger: Rechtsgeschäfte über das Droit moral im deutschen und französischen Urheberrecht. Nr. Heft 41 in Urheberrechtliche Abhandlungen des Max-Planck-Instituts für ausländisches und internationales Patent-, Urheber-und Wettbewerbsrecht. Verlag C. H. Beck.

(Mollenhauer/Blees/Rittberger) Luca Mollenhauer; Ingo Blees; Marc Rittberger: Open Educational Resources (OER) in Deutschland fördern. Der Diskurs und die Bildungspolitik zu Open Educational Resources in Deutschland am Beispiel des Förderprogramms OERinfo. Information - Wissenschaft und Praxis, 68(2-3):179-181, 2017.

(Moscon 2015) Valentina Moscon: Academic freedom, copyright, and access to scholarly works: A comparative perspective. In: R. Caso; F. Giovanella (Hrsg.), Balancing copyright law in the digital age: some comparative perspectives. Springer.

(Ostrom et al. 2008) Elinor Ostrom; Roy Gardner; James Walker: Rules, games, and commonpool resources. The University of Michigan Press.

(Ostrom 1990) Elinor Ostrom: Governing the commons. The evolution of institutions for collective action. Cambridge University Press, 20. (2007) Aufl.

(0`Neill 2005) Johnathan O’Neill: Originalism in American Law and Politics. A Constitutional History. The Johns Hopkins University Press.

(Parthey/Umstätter 2002) Heinrich Parthey; Walther Umstätter (Hrsg.): Wissenschaftliche Zeitschrift und Digitale Bibliothek. Wissenschaftsforschung Jahrbuch 2002. Gesellschaft für Wissenschaftsforschung - https://bit.ly/2mcol5A.

(Peifer 2020) Karl-Nikolaus Peifer: Anpassungsbedarf durch die neue Urheberrechtsrichtlinie. GRUR, S. 14-22, 2020.

(Peifer 2019) Karl-Nikolaus Peifer: Die urhebervertragsrechtlichen Normen in der DSM-Richtlinie. ZUM, S. 648 ff., 2019.

(Peifer 2015) Karl-Nikolaus Peifer: Festhalten am idealistischen Schöpferbegriff?: In: (Dreier/Hilty 2015), S. $351 \mathrm{ff}$.

(Peukert 2018) Alexander Peukert: Kritik der Ontologie des Immaterialgüterrechts. Geistiges Eigentum und Wettbewerbsrecht 134. Mohr Siebeck.

(Peukert 2017) Alexander Peukert: Kommentar zu §38,4, RN 45-47. In: Schricker; Loewenheim (Hrsg.), Urheberrecht. C. H. Beck, 5., neu bearbeitete Aufl.

(Peukert 2012b) Alexander Peukert: Das Verhältnis zwischen Urheberrecht und Wissenschaft: Auf die Perspektive kommt es an! JIPITEC, 1, para:142 - https://bit.ly/2nh0LKm.

(Peukert 2012a) Alexander Peukert: Die Gemeinfreiheit. Mohr Siebeck.

(Peukert 2002) Alexander Peukert: Rezension von (Metzger 2002). UFITA, 2002/III:883-887.

(Pflüger 2016) Thomas Pflüger: Die Bildungs- und Wissenschaftsschranke - Reflexionen und Überlegungen aus Sicht der Kultusministerkonferenz. ZUM, S. 484-488, 2016.

(Pflüger 2010) Alexander Peukert: Positionen der Kultusministerkonferenz zum Dritten Gesetz zur Regelung des Urheberrechts in der Informationsgesellschaft - „Dritter Korb«. ZUM, S. 938-945, 2010.

(Pflüger/Ertmann 2004) Thomas Pflüger; Dietmar Ertmann: E-Publishing und Open Access Konsequenzen für das Urheberrecht im Hochschulbereich. ZUM, S. 436-443, 2004. 
(Pflüger/Hinte 2018) Thomas Pflüger; Oliver Hinte: Das Urheberrechts-WissensgesellschaftsGesetz aus Sicht von Hochschulen und Bibliotheken. ZUM, S. 153-161, 2008.

(Praxisleitfaden 2017) Praxisleitfaden zum Recht bei E-Learning, OER und Open Content. Oktober 2017 (im Auftrag des und in Zusammenarbeit mit dem Multimedia Kontor) - https: //bit.ly/2BB3506.

(Raue 2019) Benjamin Raue: Rechtssicherheit für datengestützte Forschung. ZUM, S. 684-693, 2019.

(Reda 2019) Julia Reda: EU-Urheberrechtsreform: Der Kampf war nicht umsonst. Reda-Website, 17.4.2019 - https://bit.ly/2IClzeH.

(Reichman/Okediji 2012) J. H. Reichman; R. L. Okediji: When copyright law and science collide: Empowering digitally integrated research methods on a global scale (Review). Minnesota Law Review, 96(4):1362-1480.

(Reschke 2010) Johannes Reschke: Die verfassungs- und dreistufentestkonforme Auslegung der Schranken des Urheberrechts zugleich eine Überprüfung von §52b UrhG: Band 27 von Schriften zum und Immaterialgüterrecht. V\&R unipress.

(Rettling 2013) Falk Rettling: Die öffentlichen Kosten wissenschaftlicher Publikationen in Österreich. Version 1.0. Zenodo. 10.5281/zenodo.18338, 2013 - http://bit.ly/2xU5SBH.

(Riis/Schovsbo 2010) Thomas Riis; Jens Schovsbo: Extended collective licenses and the Nordic experience - It's a hybrid but is it a VOLVO or a lemon? Columbia Journal of Law \& the Arts, 33(4):471-498 - https://bit.ly/2pNUOpL.

(Risse-Kappen/Sikkink 1999) Thomas Risse; Kathryn Sikkink: The socialization of international human rights norms in domestic practices. Cambridge University Press DOI - https://bit.ly/ 2ThAt8c.

(Ritter 1972) Joachim Ritter: Artikel Ethik. In: Ders (Hrsg.), Historisches Wörterbuch für Philosophie, Band 2, S. 759-795. Schwabe \& Co.

(Rose 2003) Carol M. Rose: Romans, roads, and romantic creators: Traditions of public property in the information age. Law and Contemporary Problems, S. 89-110 - https: //bit.ly/2nWosbl.

(Rosenkranz 2016) Frank Rosenkranz: Anmerkung zu EuGH, Urteil vom 12.11.2015 - C-572/13 Reprobel. ZUM, S. 160 ff., 2016.

(Rubin 2012) Zick Rubin: Let's spread the word about fair use. The Chronicle of Higher Education, 23.9.2012 - https://bit.ly/31UloLn.

(Sandberger 2017) Georg Sandberger: Die Zukunft wissenschaftlichen Publizierens. Open Access und Wissenschaftsschranke. Anmerkungen zu den Kontroversen über die Weiterentwicklung des Urheberrechts. Ordnung der Wissenschaft, S. 75-95, März 2017.

(Sattler 2009) Susen Sattler: Der Status quo der urheberrechtlichen Schranken für Bildung und Wissenschaft. Eine Untersuchung anhand der konventions- und europarechtlichen sowie der verfassungsrechtlichen Vorgaben. Nomos.

(Schack 2017) Haimo Schack: Das neue UrhWissG - Schranken für Unterricht, Wissenschaft und Institutionen. ZUM, S. 802-808, 2017.

(Schack 2016) Haimo Schack: Urheberrechtliche Schranken für Bildung und Wissenschaft. ZUM, S. 266-284, 2016.

(Schack 2015) Haimo Schack: Urheber- und Urhebervertragsrecht. Mohr Siebeck, 7. Aufl.

(Schimmer/Geschuhn/Vogler 2015) R. Schimmer; K. K. Geschuhn; A. Vogler: Disrupting the subscription journals' business model for the necessary large-scale transformation to open access. doi:10.17617/1.3. 
(Schmiede 2013) Rudi Schmiede: Wissenschaft+digitale Publikation. Inetbib, 26.4.2013 https://bit.ly/2nDc6EG.

(Scholz 2018) Anna-Lena Scholz: Wem gehört das Wissen? ZEIT-Online. DIE ZEIT, Nr. 31/2018 https://bit.ly/2RmuvB4.

(Schönfelder 2019) Nina Schönfelder: Transformationsrechnung. Mittelbedarf für Open Access an ausgewählten deutschen Universitäten und Forschungseinrichtungen. Ein Bereich des National Open-Access-Kontaktpunkts-OA2020-DE - https://bit.ly/2PPA1Na.

(Schüller-Zwierlein/Leiwesmeyer 2018) André Schüller-Zwierlein; Barbara Leiwesmeyer: Neuerungen im Urheberrecht. Stand und Perspektiven. Präsentation. Universitätsbibliothek Regensburg - https://bit.ly/2HnnYld.

(Schwarz 2018) Tim Jonathan Schwarz: Die Beendigung urheberrechtlicher Nutzungsrechte: Eine dogmatische Analyse. Nomos.

(Senftleben 2004) Martin Senftleben: Copyright, limitations and the three-step test: An analysis of the three-step test in international and EC copyright law. Kluwer Law International.

(Senftleben 2010) Martin Senftleben: The international three-step test: A model provision for EC fair use legislation. JIPITEC, S. 67-82, 2010 - https://bit.ly/2nmo3hR.

(Siegmann 2006) Richard Siegmann: Über die Ketten der Wissensgesellschaft. Der Kulturkampf über den Zugang zu wissenschaftlichen Veröffentlichungen verschärft sich. c't Magazin für Computertechnik, 29.5.2006 - https://bit.ly/31uYmL5.

(Spindler 2008) Gerald Spindler: Reform des Urheberrechts im „Zweiten Korb”. NJW, 9, 2008.

(Staab 2019) Philipp Staab: Digitaler Kapitalismus in der Ökonomie der Unknappheit - Edition suhrkamp.

(Stang 2017) Felix Stang: Art. 14 der neuen DSM-Richtlinie. ZUM, S. 668-674, 2019.

(Steffen 2010) Till Steffen: Diskussionspapier „Nutzerorientierte Ausrichtung des Urheberrechts".

(Steinhauer 2017) Eric W. Steinhauer: Die Urheberrechtsmärchen der FAZ zum geplanten UrhWissG. Kapselschriften, 19.5.2017 - https://bit.ly/33VppBb.

(Steinhauer 2014) Eric W. Steinhauer: EuGH-Urteil zu elektronischen Leseplätzen stärkt Wissenschaftsurheberrecht. GRUR-Prax, S. 471-473, 2014.

(Steinhauer 2013) Eric W. Steinhauer: Mehr Open Access oder bloßer Placebo? Legal Tribute Online, 23.9.2013 - https://bit.ly/2mchz5f.

(Steinhauer 2010) Eric W. Steinhauer: Das Recht auf Sichtbarkeit. Überlegungen zu Open Access und Wissenschaftsfreiheit. Verlagshaus Monsenstein und Vannerdat OHG - https: //bit.ly/2IFLBhi.

(Stemmer 2011) Peter Stemmer: Die Konstitution der normativen Wirklichkeit: In: (Forst/Günther 2011), S. 57-68.

(Stieper 2009) Malte Stieper: Rechtfertigung, Rechtsnatur und Disponibilität der Schranken des Urheberrechts. Mohr Siebeck.

(STM 2018) The STM Report. Fifth edition. Rob Johnson and Anthony Watkinson and Michael Mabe (Hrsg.): An overview of scientific and scholarly publishing - https://bit.ly/2PpIVOo.

(Straumsheim 2016) Carl Straumsheim: How to measure impact. Inside Higher ED vom, 14.12.2016 - https://bit.ly/36cnfy1.

(Surman 2019) Mark Surman: Simple Geschichten über KI sind eine Gefahr. Tagesspiegel Background, 8.5.2019 - https://bit.ly/3cow0bq.

(Talke 2019) Armin Talke: Warum die Urheberrechtsreform die Bibliotheken betrifft. BLOGNETZWERK FÜR FORSCHUNG UND KULTUR der Staatsbibliothek zu Berlin, 22.1.2018 https://bit.ly/2Gy2pjb. 
(Taubert 2019) Christian Taubert: Fremde Galaxien und abstrakte Welten. Open Access in Astronomie und Mathematik: Eine soziologische Analyse. transcript - https://bit.ly/2mnRWhO. Preprint 2017: https://bit.ly/2nQDG1m.

(Teach Act 2011) The Teach Act, New roles, rules and responsibilities for academic institutions. Copyright Clearance Center - https://bit.ly/2m2PZqt.

(Tiersma 2010) Peter M. Tiersma: Parment, paper, pixels. Law and and the technologies of communication. The University of Chicago Press.

(Till et al. 2019) Brian M. Till et al.: Who is pirating medical literature? A bibliometric review of 28 million Sci-Hub downloads. Correspondence, 7, 1.1.2019 - https://bit.ly/2EvW32M.

(Triaille et al. 2013) Jean-Paul Triaille and others (De Wolf \& Partners): Study on the application of Directive 2001/29/EC on copyright and related rights in the information society - https: //bit.ly/2m5iDY9.

(Turowski/Mikfeld 2013) Jan Turowski; Benjamin Mikfeld: Gesellschaftlicher Wandel und politische Diskurse. Überlegungen für eine strategieorientierte Diskursanalyse. Denkwerk Demokratie. Hans Böckler Stiftung September 2013 - https://bit.ly/2moKzXs.

(Ulmer 1951/1960) Eugen Ulmer: Urheber und Verlagsrecht. Springer.

(von Ungern Sternberg 2019) Joachim von Ungern-Sternberg: Die Herausgeberbeteiligung der VG WORT - rechtswidrige Ausschüttungen an nichtberechtigte Dritte. urPC Web-Dok. 25/2019, Abs. 1-74 - https://bit.ly/2IDpsQG.

(Vaihinger 1911) Hans Vaihinger: Die Philosophie des Als Ob. System der theoretischen, praktischen und religiösen Fiktionen der Menschheit auf Grund eines idealistischen Positivismus. Mit einem Anhang über Kant und Nietzsche. Reuther \& Reichard.

(Völtz 2011) Gregor Völtz: Die Werkwiedergabe im Web 2.0. Reformbedarf des urheberrechtlichen Öffentlichkeitsbegriffs. Gabler.

(Walger/Walger 2019) Nicole Walger; Nadine Walger: Vom Schein des Rechten getäuscht. Raubverlage und was die Wissenschaft dagegen unternehmen kann. Information, Wissenschaft \& Praxis, 2-3:91-97, 2019.

(Wandtke 2017) Artur-Axel Wandtke: Urheberrecht. De Gruyter, 6. Aufl.

(Wandtke/Hauck 2019) Artur-Axel Wandtke; Ronny Hauck: Art. 17 DSM-Richtlinie - Ein neues Haftungssystem im Urheberrecht. ZUM, S. 627 ff., 2019.

(Wandtke/Bullinger 2014) Artur-Axel Wandtke; Winfried Bullinger: Praxiskommentar zum Urheberrecht. C. H. Beck, 4. neu bearbeitete Aufl.

(Wandtke/Grassmann 2006) Artur-Axel Wandtke; Ferdinand Grassmann: Einige Aspekte zur gesetzlichen Regelung zum elektronischen Kopienversand im Rahmen des »Zweiten Korbs«. ZUM, S. 889 ff., 2006.

(Weber 2017) Hubertus Weber: Die urheberrechtliche Zwangslizenz. Erweiterte kollektive Lizenz. Nomos/Stämpfli.

(Wefing 2014) Heinrich Wefing: Lex Snowden: Europas Richter schützen die Daten der Bürger vor dem Staat. Aber hilft das gegen die Sammelwut der Amerikaner? DIE ZEIT, (Nr. 16):1, 10.4.2014 - http://bit.ly/1jdyYQp.

(Wildgans 2019) Julia Wildgans: Zuckerbrot oder Peitsche? - Ein Plädoyer für Open Access im juristischen Publikationswesen. ZUM, S. 21-28, 2019.

(Wille 2009) Stefan Wille: Anmerkung zu BVerfG, Beschluss vom 24. November 2009 - 1 BvR 213/08. ZUM, S. 240-243, 2010.

(Wipo 2018) World intellectual property indicators 2018 WIPO. Genua.

(Wissen 2004) N. N: Wissen. In: Joachim Ritter; Karlfried Gründer; Gottfried Gabriel (Hrsg.), Historisches Wörterbuch der Philosophie, Band 12 (W-Z). Schwabe AG Verlag. 
(Wissenschaftsrat 2001) Wissenschaftsrat: Empfehlungen zur digitalen Informationsversorgung durch Hochschulbibliotheken, 13.7.2001 (Drs. 4935/01) - https://bit.ly/2P5Tbz).

(Witschen 1998) Dieter Witschen: Was verdient moralisch den Vorzug? Ethisches Abwägen durch Präferenzregeln. Verlag W. Kohlhammer.

(Wittem Group 2010) Wittem Group: The Wittem Project. European copyright code. April 2010 www.copyrightcode.eu.

(Ziegler 2019) Günther M. Ziegler: Die Bedeutung der Verlage ändert sich. Forschung \& Lehre, 8.6.2019 - https://bit.ly/2nl8RS4.

(Zippelius 2004) Reinhold Zippelius: Verhaltenssteuerung durch Recht und kulturelle Leitideen. Duncker \& Humblot.

(Zoglauer 1998) Thomas Zoglauer: Normenkonflikte - Zur Logik und Rationalität ethischen Argumentierens. Friedrich Frommann Verlag \& Günther Holzboog.

(Zott 2002) Regine Zott: Der Brief und das Blatt. Die Entstehung wissenschaftlicher Zeitschriften aus der Gelehrtenkorrespondenz: In: (Parthey/Umstätter 2002), S. 47 ff. 